\title{
Transformation of the personnel competency model in the context of the transition to the digital economy
}

\author{
Anna Belolipetskaya ${ }^{1}$, Tatyana Golovina ${ }^{1}$, Andrey Polyanin ${ }^{1}$, and Yulia Vertakova, ${ }^{2, *}$ \\ ${ }^{1}$ Central Russian Institute of Management, Branch of RANEPA, 5A, Boulevard Pobedy, Orel, \\ 302028, Russian Federation \\ ${ }^{2}$ Southwest State University, 94, 50 Let Oktyabrya str., Kursk, 305040, Russian Federation
}

\begin{abstract}
The purpose of the research is to study the problems of the competence interface of personnel management of business structures in the context of digital transformation and to justify the organizational and managerial measures for its development and effective use. The analysis of various approaches to highlighting the competencies of personnel in the digital economy, by which is meant the confident and selective use of information and communication technologies in the ecosystem, is presented. It is determined that the use of digital technologies is a prerequisite for ensuring the competitiveness of business structures. The leading role of "soft" skills, including both cognitive and socio-behavioral abilities, is substantiated. A three-module model of personnel competencies in the context of digital transformation is suggested, in which such digital competencies and skills as: working with digital technologies, creative thinking and behavior, professionally oriented skills in the field of production and managerial activities are highlighted. The use of the competency-based approach to the digital transformation of economic processes helps to increase the adaptability of the existing system of economic relations to the conditions of the new technological structure, and provides the possibility of introducing end-to-end technologies into management decision-making processes in order to increase their efficiency.
\end{abstract}

\section{Introduction}

Classical economic theory is gradually being transformed into the economics of information, which is a collection of ideas and texts. This scientific direction has not formed instantly and has its own history. Many prominent academics stood at the origins of the economics of information, including J. Marshak, J. Stigler, J. Stiglitz, J. Akerlof and other researchers $[1,2]$. Their works can be considered the beginning of the process of establishing a new economic paradigm.

For digital transformation, the development of information technologies (digitalization) is not enough, the restructuring of business processes, the new organization of working with

* Corresponding author: vertakova7@yandex.ru 
data, the formation of new business models and strategies, the training and adaptation of people, the formation of a digital culture and digital society are necessary. It is important that these processes are interconnected, and the potential of an organization should be considered in the context of the ecosystem.

It should be noted that the economy itself should not be defined on the basis of any external form of its organization: virtual, digital, informational or any other. Meanwhile, the formulation of the most accurate and comprehensive definition of it should meanwhile reflect specific goals, directions of development and the nature of the results, which can also vary significantly and create risks of reducing economic security from objective national economic to non-constructive private egocentric.

In the era of digital business transformation, business entities need more competencies. According to T. Casas, professor of international business, «Digitalization is not only about technology, but also about business models and internalization». E. Kaganer notes that "Transformation of a company comes down to the transformation of people's competence. $\mathrm{CDO}$ is not a profession, it is a role. A role of a person who combines many competencies and skills» [3].

The competency-based approach to personnel management has its own history of formation, which is closely related to such names as D. McClelland, L. Spencer, R. Boyatzis and White [4,5]. They have come to the conclusion that taking academic assessments into account and using intelligence tests is not enough to identify an effective successful potential employee of a company.

An obvious similarity is that the authors in almost all definitions emphasize the importance of competence primarily for effective work in a certain position: the effective implementation of specific tasks within a given organization, the effective use of internal and external resources for setting and achieving goals.

At the same time, E. Short's research focuses on flexibility, adaptability, the ability of an individual to actively respond to the impact of the environment [6]. In the definition of O.E. Yarygin competence is considered as an area of problems to be solved, a circle of specific duties of an employee [7]. G.K. Selevko claims that competence is primarily willingness and ability of an employee to organize external and internal resources in the best way to achieve their goal [8].

In the work of K. Ala-Mutka it is defined that the essence of competence is consistent with the European Qualifications Framework (EQF), which in turn includes Knowledge - a set of principles, theories and practices learned in the educational process, Skills - the ability to use knowledge and know-how as solutions to various problems, Attitudes - these are such components as motivation, responsibility, autonomy [9].

It is worth agreeing with the author's conclusion that competencies are a component of the highest level, including knowledge, skills and how they are applied in practical aspects. At the same time, K. Ala-Mutka does not give due consideration to the combinations and variations of the application of the concept of competencies and equates the concepts of competence and skills.

In this context, substantiated conclusions are contained in the works of C. Iordache, I. Marien, D. Baelden, who note that knowledge is a prerequisite for the formation of skills and the main thing in their difference is the "relational" aspect [10].

It should be noted that the concept of digital literacy was first popularized by P. Gilster in his book in 1997. The researcher understands "digital literacy" as "the ability to critically understand and use information" that is obtained through a computer. Obviously, in connection with the rapid spread of the Internet, the use of this term has become widespread and in demand [11]. In turn, A. Martin managed to concretize "digital literacy", which includes "awareness, attitudes and the ability of individuals to properly use digital instruments and tools" to manage or evaluate digital resources [12]. 
Thus, both foreign and Russian scientists pay much attention to the problems of the formation of digital competencies, the organization of work of the professional community in order to develop information systems, the introduction of innovative technologies and services in the healthcare sector. The works of many scientists and professional communities are devoted to the study of the processes of the formation of new technological structures, the introduction of digital technologies in the economic and social sphere.

Experts most often consider the organizational and technical aspects of the use of new technologies, assess the probability of human labor being replaced by them: Vidas-Bubanja M., Taylor E., Brynjolfsson, Acemoglu, Boston Consulting Group. etc. [13,14,15].

Scientists from the University of Oxford Carl Frey и Michael Osborne have presented one of the most critical predictions for the study of a paradigm shift in the competencybased approach to digital transformation processes [12]. According to their estimates, the gap between the level of existing digital skills and the capabilities of end-to-end technologies will widen in the next five to ten years.

At the same time, in existing publications and scientific developments, the issue of digital transformation and its staffing is considered mainly as a whole, in some cases with an emphasis on business structures, without studying the problems of creating a competency-based model of a digital ecosystem in relation to the national economy.

The experts predict that $[13,14]$, deficit in such areas as artificial intelligence, nanotechnology, 3D printing and other technologies, the appearance of which is associated with a new era, which will radically change the pattern of consumption, production and employment, will add to the existing digital gap. Thus, it is the level of digital competencies of personnel that will determine the success of digitalization processes.

According to the authors, "digital competence" is a sophisticated and complex phenomenon that determines the life of a person in various areas of the information society.

It is generally accepted to determine the following components in the structure of "digital competence": skills; knowledge; motivation; responsibility. Any component of "digital competence" is implemented on the Internet in various fields of activity, for example, communication, technosphere, work with content, etc.

In this regard, the following types of "digital competence" are identified in the scientific world:

1) "Information and media competence" that includes knowledge, skills, motivation and responsibility;

2) "Communicative competence" that includes knowledge, skills, motivation and responsibility;

3) "Technical competence" that includes knowledge, skills, motivation and responsibility;

4) "Consumer competence" that includes knowledge, skills, motivation and responsibility; [3].

The purpose of the research is to study the problems of the competence interface of personnel management of business structures in the context of digital transformation and to justify the organizational and managerial measures for its development and effective use.

\section{Problem Statement}

The world community does not currently have a unified approach to highlighting the competencies of the digital economy, which makes it difficult to build a competency model in Russia taking into account digitalization processes. The authors consider it appropriate to undertake a critical assessment of the most common competency-based approaches.

According to the World Bank Global Digital Development Report "Digital Dividends," 
all the skills demanded in the digital economy can be divided into three groups:

- cognitive;

- social and behavioral;

- technical [14].

Analytics of the World Economic Forum believe that in the next five years, more than a third of the skills that are currently considered important in professional work will change. They include: critical thinking, creativity, ability to work with people, customer-orientation [15].

In turn, DigComp offers to form a model of digital competencies from five areas, namely: Information and Data Literacy Competence, Communication and Collaboration Competence, Digital Content Creation Competence, Safety Competence, Problem Solving [14].

The authors' point of view is that the advantage of this model is the availability of detailed grading scales for eight qualification levels of EQF.

However, this system is based precisely on the use of digital technologies and does not cover such important points as cognitive and personal qualities, value attitudes.

The model of fundamental digital skills proposed by Burning Glass merits the attention. In this model four blocks are highlighted: Human Skills, Domain Knowledge, Digital Building Block Skills, Business Enabler Skills, and also the levels of development of digital competencies are defined [16].

Thus, in foreign concepts, an integrated approach to competencies in the management of human resources is used, which "contains both behavioral and functional approaches and includes cognitive, behavioral and functional competencies in an integrated structure."

The holistic model of competencies consists of the following types of competencies: cognitive, functional, social and meta-competencies.

1) cognitive competencies contain both formal and informal knowledge, based on experience.

2) functional competencies include the skills that an employee that carries out labor activities in a specific professional field, should possess.

3) social competencies consist of personal and ethical competencies.

4) meta-competencies refer to the ability to cope with uncertainty, teachings and criticism.

One can consider the emphasis on digital skills, which are not enough for the successful development of an employee in modern conditions, to be the disadvantage of these approaches.

The analysis shows that there is a large classification of digital competencies for various reasons, which plays the most important role in their practical use.

1) In terms of distribution, the distinction is made between general competencies necessary for all positions of a given organization and specific competencies that are characteristic of representatives of a particular profession or group of professions in a given organization. Within the framework of general competencies, it is possible to identify managerial competencies that should be owned by representatives of this profession, whose activities are directly related to planning, organization, management and control.

2) By the essence and content, one can identify the competencies necessary for effective work and the competencies associated with individual effectiveness. The first ones include cognitive competencies related to knowledge and understanding, as well as functional competencies based on applied skills, and competencies related to individual effectiveness include personal (self-development, motivation) and social competencies (behavior and relationships).

3) According to the level of development, threshold and differentiating competencies are distinguished. Threshold competencies are essential characteristics for starting an 
activity. Differentiating competencies distinguish the best performers from medium ones. Specialists identify the following types of competencies: corporate, managerial and professional (technical). Corporate competencies can be applied to any position in the organization.

It is important to understand that the development of an organization involves a change in the requirements of the employee; therefore, it is necessary to allow and adhere to these requirements that emerge over the course of this development. Personnel assessment is applied for it. End-to end assessment involves identifying correspondence between a person's competencies and competencies which are currently needed in the organization. End-to-end assessment is divided by frequency into: current, monthly, quarterly and annual. Current assessment is carried out by the line manager weekly on the score sheets (or in electronic form), where he describes the employee's activities regarding the performance of the prescribed functions, labor behavior and the employee's attitude to work.

The main purpose of current assessment is to monitor labor behavior and respond to all changes quickly. Monthly assessment implies a manager's assessment of the employee's performance of tasks within a month in order to determine the form of monetary and nonmonetary compensation.

The authors suggest considering the following components of personnel assessment:

1. The competence of ethical behavior. Knowledge, observance of professional ethical values and intolerance of non-observance of these values.

Is not properly developed: the difference from the values formed in the organization, establishes their own rules, excessive individualism, can neglect reflections on their own values and does not think about the impression $\mathrm{s} / \mathrm{he}$ makes.

Is developed: Adheres to an effective and appropriate set of values (for the collective) both in the worst and in the best of times. Actions correspond to these values. Notices the promotion of these values and notes any inconsistencies.

Is excessive: Adheres to and defends their values or the position associated with them, when it is not appropriate. Sensitive to situations of verification of principles. May gain a reputation as stubborn and unresponsive when refusing to compromise.

2. Creativity concept. The ability to organize work, the ability to work creatively, independence.

Is not properly developed: Narrow-mindedness, introversion, prudence, conservatism, wariness.

Is developed: Suggestion of many unique and new ideas. Capable of finding a connection between disparate representations. The reputation of the idea generator in brainstorming.

Is excessive: Losing time thinking about a secondary task. Not bringing ideas to implementation. Disorganization and / or inattention to details. Individualism or selfish behavior when working with a collective.

3. Competence of analytical work. The ability to assess the quality and completeness of knowledge, the formation of a knowledge base. Structuring information. Identification of new knowledge through research activities. Application of this knowledge.

Is not properly developed: Solving new problems with old methods. Limited qualifications. Persistence in decision making without sufficient justification. Complex structuring of information. Lack of desire to identify new knowledge.

Is developed: Capable of finding a connection between disparate representations. The thoroughness, complexity and duration of projects. The workflow is shared, goals and objectives are set. Sees the interconnection of various elements. Knows the strategy of work and tactics in their field.

Is excessive: Disorganization and / or inattention to details. Filling in the gaps with fictional facts. Excessive complexity of reality. 
4. Organizational skills. Finding and making organizational management solutions.

Is not properly developed: Ineffective organization and use of resources. Does not know how to delegate authority and stimulate to work. Ineffective work by solving two cases at the same time. Excessive self-confidence. Postponement of tasks until the last moment of the deadline.

Is developed: Optimal organization and use of resources. Leading of various activities to achieve the goal. Structuring information for the ease of use.

Is excessive: Cannot tolerate disorder. Does not allow the deviation of the workflow from the familiar process. Does not accept suggestions and new information. Not effective if everything went wrong.

5. Competence of planning. Planning of events in accordance with the general development strategy of the region and the state.

Is not properly developed: May be one of the workers who does everything at the last minute. Neglectful of planning. May experience a sense of comfort when structuring the work process. Does not have the patience necessary for planning deadlines, completing work, setting goals and objectives. Demotivation of the collective.

Is developed: Foresees problems. Responsibly treats planning, studies the task, reveals the complexity of the task and in this regard plans their activities. Engaged in setting goals and objectives. Matches implementation with purpose. Capable of assessing the result.

Is excessive: Too strict adherence and dependence on the rules. Not taking into consideration the human factor. Not adaptable to rapid change.

6. Decision making competence. Developing solutions basing on the regulatory and legal framework.

Is not properly developed: Makes hasty decisions without analyzing the situation. Excessive self-confidence. Inability to choose the right methods and solutions. Shortsightedness and prejudice. Spending too much time working on small things to avoid risks. Work on a extensive solution, instead of considering small ones.

Is developed: Making good decisions based on analysis, knowledge and experience, without thinking about the duration of the implementation. Most decisions turn out to be correct and accurate. Has the authority of a person to whom one can turn for advice.

Is excessive: Inability to listen to others because of their "always right decisions", as if $\mathrm{s} /$ he has never been never wrong. Inability to compromise, stubbornness.

Under the current circumstances, the digital economy is beginning to take an active position in all spheres of social life, having a significant impact on the labor market. Digitalization of the economic system is the main direction of development of the state, economy and public relations, and the digital economy, in turn, is becoming a breakthrough technology in the system of sustainable development of society. Economic science and education in the new conditions cannot develop according to the traditional scenario; their transformation is inevitable taking into account existing realities.

Digital transformation has already affected a quarter of the global economy. Its main task is to radically increase efficiency, raise industries and countries. Digital transformation is changing traditional business models; according to it completely new players appear in established areas of the economy. The digital transformation of the business, aimed at increasing the speed of decision-making, increasing the variability of processes and increasing their efficiency, is based not only on the desire of companies to meet customer expectations, but also on the adaptation to rapid changes in the world [17]. The digital economy is providing fewer opportunities to search for new markets for a consistent value proposition. Digital technologies have eliminated many barriers to companies entering the market, so it is likely that an existing offer has already reached all possible customers. Any change in consumer demand, the appearance of new products or many substitute products entering into the market may lead to a reduction in the market. Correspondence of the value 
proposition to the new conditions, aimed at ensuring that the company becomes in demand for customers, involves the use of customer-oriented strategies, which are focused on outstripping environmental fluctuations If a company is able to revise the proposed sets of values, identify new customer requests, notice emerging opportunities, it looks to the future. The creation of popular products and services in the context of constant changes in external conditions is based on the using of data and turning it into useful information.

According to the IDC and Microsoft study "The State of Digital Skills in Central and Eastern Europe", in which enterprises from Russia also took part, today it is already necessary to develop a model of digital competencies of personnel [13].

The study was conducted in 2019 in six countries of Central and Eastern Europe: the Czech Republic, Greece, Hungary, Poland, Romania and Russia. This was a quantitative survey of more than 700 organizations with more than 10 employees, with a representative vertical sample for each country. Respondents represented various managerial and organizational levels and had the authority to make decisions, or at least acted as consultants in making decisions regarding training and professional development of employees of their organizations.

According to the respondents, at the moment, among the most relevant there are digital skills in the field of automation and robotization of processes, mobility, big data analysis, IT architecture and the transition to new architectures, as well as networking. But in 2-3 years, the needs of the business will become different, and therefore the list of skills, the demand for which will be especially high, is going to change. First of all, the skills in Big Data analysis will come to the fore. However, the skills in collaboration applications will take the second place, as predicted by business representatives. Skills in the field of automation and robotization of processes will not disappear from the top, but they will move from the first place to the third. The survey results indicate that in the near future qualified IT professionals who can widely use digital systems and applications and learn fast will be the most valuable employees. At the same time, about two-thirds of respondents believe that in the next 2-3 years they will need more flexible specialists of a wide profile. Further in the ranking of potential demand there are employees with basic IT skills and creators of advanced technological tools (Figure 1).

At the same time, today, in more than half of cases, the digital skills of employees meet the requirements of employers on an average level. And only $3.6 \%$ of employees are fully in line with the digital skills demands of employers (Figure 2).

It is important to note that according to the survey participants, $80 \%$ of companies in Russia are at the initial stage of digital transformation. Approximately the same proportion of responses was recorded in Greece and Hungary. However, the share of those who assessed the state of digital transformation as being at an advanced stage in Russia is more than $15 \%$ - according to this indicator, our country is inferior only to Romania, where it is 19.3\% (Table 1). 


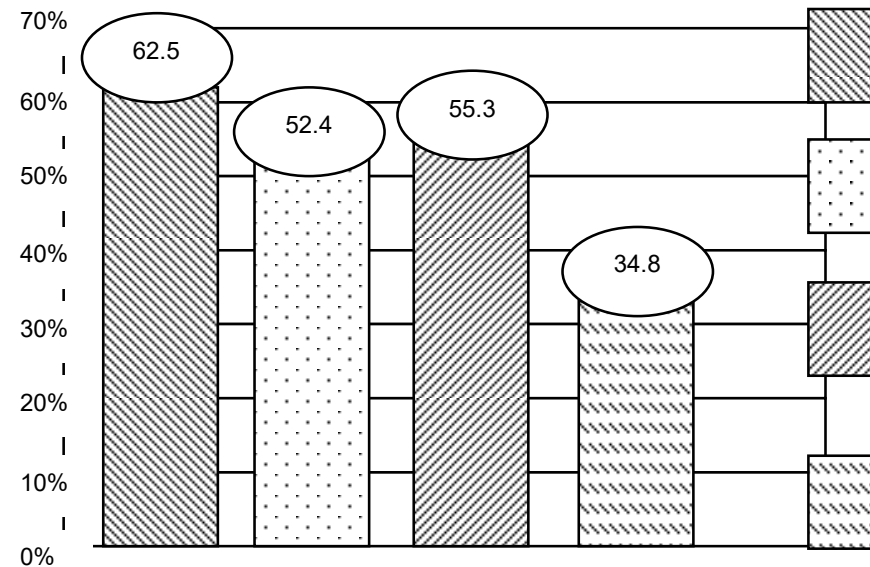

IT - specialists

technology producers

candidates with basic IT skills

employees without IT qualifications

Fig. 1. Demand for digital skills in Central and Eastern Europe, \% [13]
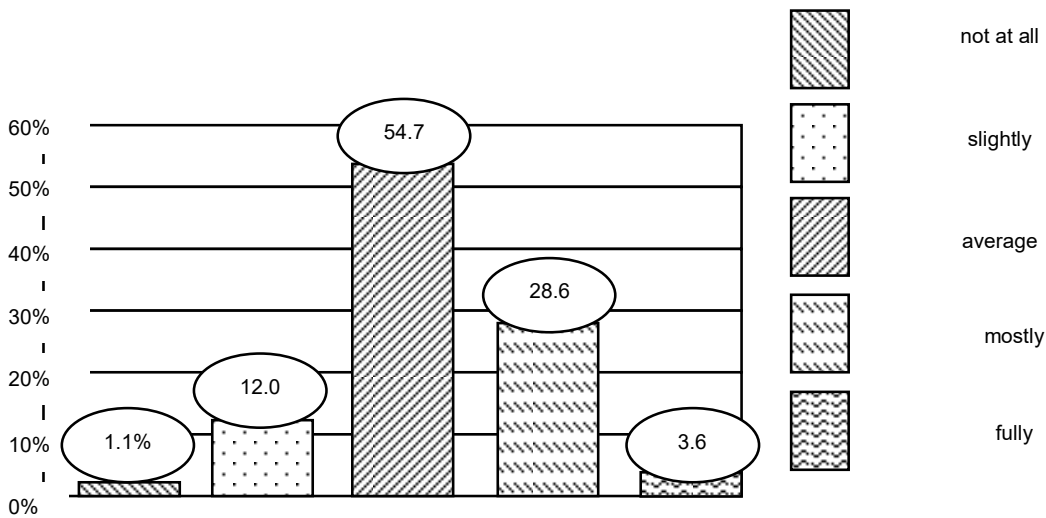

Fig. 2. Digital skills preparedness in Central and Eastern Europe, \% [13]

Table 1. State of digital transformation in Central and Eastern Europe, \% [13]

\begin{tabular}{|c|c|c|c|c|c|c|}
\hline & $\begin{array}{c}\text { The Czech } \\
\text { Republic }\end{array}$ & Greece & Hungary & Poland & Romania & Russia \\
\hline $\begin{array}{c}\text { The } \\
\text { advanced } \\
\text { stage }\end{array}$ & 11.4 & 11.1 & 8.2 & 11.8 & 19.3 & 15.5 \\
\hline $\begin{array}{c}\text { The initial } \\
\text { stage }\end{array}$ & 59.1 & 81.5 & 81.8 & 79.4 & 73.4 & 80.7 \\
\hline $\begin{array}{c}\text { Has not } \\
\text { begun yet }\end{array}$ & 29.5 & 7.4 & 10.0 & 8.8 & 7.3 & 3.7 \\
\hline
\end{tabular}

\section{Results and Discussion}

According to the authors, competencies are characterized not only by people's professional skills, but also by the widely understood culture of a digital society, to which an overly technocratic approach should not be applied. At the same time, the basic and professional competencies formed in the system of general and vocational education should be consistent with the qualification requirements recorded in various professional standards.

Today, when forming and using competencies, the Russian economic system is facing 
the following problems:

1. Limited resources for the implementation and maintenance of digital systems;

2. Reluctance towards coordinated actions and mutually agreed initiatives to create integrated technology platforms and towards a simultaneous transition of the participants of economic processes to work with them from;

3. Under-resourced digitalization process, lack of skills in applying new technologies, incompleteness of digital competencies;

4. Ignoring customers - digital leaders, rejecting the request for digitalization from technologically advanced ecosystem participants;

5. Conservatism and the desire to "work in an old fashioned manner", in every possible way avoiding digital innovations;

6. Undeveloped digital infrastructure.

Strategies aimed at developing digital technologies require the inclusion of a specific set of skills, which include the non-digital component in the form of economic, financial and tax knowledge, often associated with relevant digital innovations. This set includes entrepreneurial skills, such as readiness to take risks, adaptability and critical thinking, and advanced digital skills such as data analysis, cloud computing, social networks, digital marketing and the development of network solutions and applications.

The creation and successful functioning of the ecosystem is impossible without people with a high level of digital literacy. At the same time, digital literacy is not a characteristic spontaneously acquired by a person born in the digital age. This is a system of knowledge, skills and attitudes, essential for life in a digital society, their formation and development should be conscious and manageable, and only under this condition the main goal of digitalization (to increase management efficiency) can be achieved.

At the same time, indicators of measuring digital literacy should be formulated based on an analysis of the objective needs of the economy; based on big data and machine learning technologies, vacancies are studied and employers' requirements for digital skills and knowledge of candidates (demand-side analysis) are typified. The competency model reflects the vision of what demand for human resources will appear in the future and which competencies require increased attention from the management in order to carry out its development, and this model also helps to plan human resources and create a personnel reserve on a long-term basis. Thanks to the competency model, the selection and assessment criteria are coordinated with the organization's strategy.

Countries that are implementing a comprehensive digital skills strategy are equipping their populations with the skills necessary to gain employment benefits, increase productivity, creativity and success, while maintaining an appropriate level of security, integrity and efficiency online. It is extremely important to regularly update digital skills strategies in response to new technologies and their impact on the digital economy and digital society. Many countries view digital skills as the foundation of digital transformation.

The demands of economic sectors are developing rapidly with the advent of new technologies, the emergence of new companies and new enterprises. This dynamic environment requires proactive responses from stakeholders - new partnerships, programs, and initiatives that include innovative approaches to connect learning and work. Having a favorable business environment is a prerequisite for promoting digital transformation, digital entrepreneurship and other opportunities arising from new digital technologies.

In this regard, the authors consider it appropriate to use the three-module model of personnel competencies in the context of digital transformation in the activities of business entities (Figure 3). 


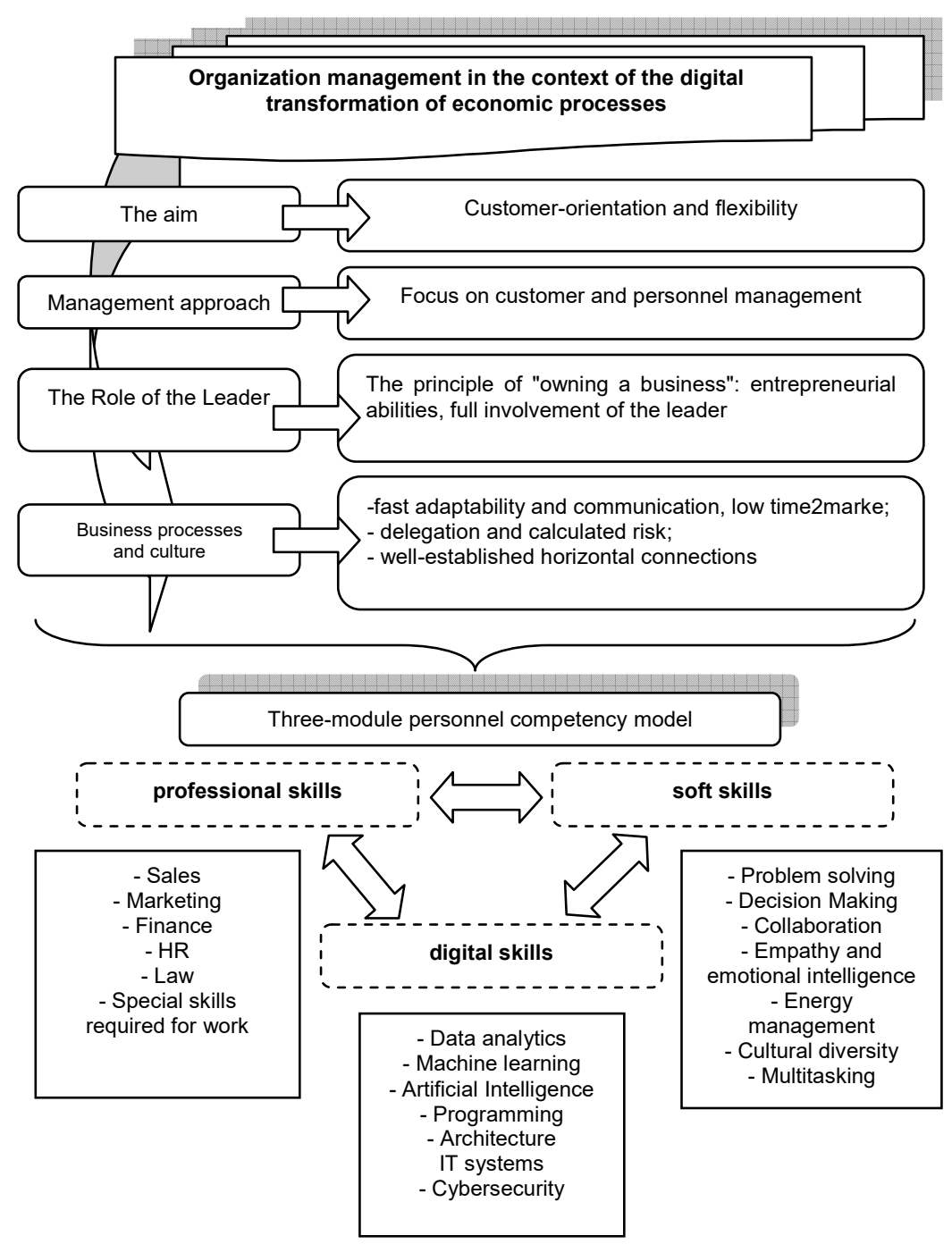

Fig. 3. Three-module personnel competency model in the context of digital transformation

The proposed three-module model of personnel competencies will allow the employee to make the best use of their strengths and talents. According to the authors, the structure of this model will require differentiated approaches to the selection and development of the personnel of the organization.

Run the business: deep expert knowledge in its functional field, executive discipline, and focus on the effective execution of tasks within the framework of established rules and standards.

Change the business: teamwork, focus on results and solving non-standard problems, self-organization, empathy, readiness for change.

Disrupt the business: willingness to take risks and experiment, tolerance for mistakes, unconventional thinking, and entrepreneurial spirit.

\section{Conclusion}

The formation of the digital contour of the ecosystem should be based on a clear 
understanding of the competency profile, its inclusion in the innovative system of conservation and development of human capital.

The authors believe that the competency-based approach to personnel management is a system that helps organizations to clearly set the parameters the employees should have and the qualities that are necessary for their future growth. The application of this method requires openness and transparency of the organization, and if it can implement it, then employees understand that this organization is predisposed to employees, sets clear requirements and rewards for fulfilling these requirements.

The authors identify four defining goals that should be realized by a successful employee in a digital environment. These include:

- Continuous learning and innovation (a quick change of scenery requires the formation of skills for quick adaptation, training and innovation);

- insights (successful technology of working with a lot of information - generalization, highlighting the main, etc. to create new ideas);

- network interaction (includes competencies in networking, teamwork, the ability to persuade and lead);

- perfection of performance (the ability to take responsibility, make bold effective decisions).

If talking about the competencies of the leader, then the environment of the digital category will present its requirements for each of them, depending on the situation and context. It is most likely that the necessary competencies will relate to productivity growth (rapid change in processes, effectiveness in conditions of uncertainty, high risks, etc.). In the global work community, digital skills not only serve as qualifications for employment in the traditional sector, but also open the door to participation in emerging sectors of the economy and even to starting one's own business. Personnel with more advanced digital skills can take advantage of the wider range of capabilities associated with the continuous development of digital technologies, platforms and devices. Digital skills are especially important in the perspective of the changing nature of the work environment, including a sharp increase in the use of labor by freelancers and people participating in the free-market economy, as well as wider structural changes that will significantly affect jobs in the future.

To remain in the workplace, a specialist should be flexible and adaptable, be prepared to change direction, constantly develop new skills and competencies, and try to do it faster than before. The set of competencies has long ceased to be static and fixed; it changes in parallel with changes in the economy, taking the form of "dynamic portfolios". Business structures should not just create staff, but create organizational stock skills, which is a set of specialists with a holistic "portfolio of competencies" necessary to complete a particular work task.

The study shows that the identification and systematization of key competencies in the context of global digitalization is at an initial stage and requires the development of public policy and of course the continuation of scientific research.

Successful implementation of the competency model in the digital economy requires new directions for the development of an appropriate organizational culture, as well as the use of digital educational technologies. Further research requires interlinking the formation of digital competencies of business entities and the strategy of digital transformation, gaps between the target and the existing level of digital maturity.

Representatives of the " $Z$ " generation are characterized by multitasking, dependence on various digital and mobile devices, interpersonal interaction to a greater extent through social networks, and the use of the Internet as the main source of information. In this regard, it is necessary to change the teaching style in the direction of developing the skills of search, analysis, processing of data arrays to obtain high-quality knowledge. 
The reported study was funded by RFBR, project number 19-310-90036

\section{References}

1. G.A. Akerlof, J.L. Yellen, Quarterly Journal of Economics. 100. Supplement. 244-259 (1985).

2. C. Ertel, L.K. Solomon, Moments of impact: How to design strategic conversations that accelerate change. (N.Y.: Simon \& Schuster, 2014).

3. The future of jobs. Employment, skills and workforce strategy for the Fourth industrial revolution / World Economic Forum, (2016). [Electronic resource]: http://reports.weforum.org/future-of-jobs-2016/ (accessed 20.01.2020).

4. F.M. Fossen, A. Sorgner IZA Discussion Paper 12242. (Bonn: Institute of Labor Economics, 2019)

5. T. Kelley, D. Kelley Creative confidence unleashing the creative potential within us all (Crown Business, 2013).

6. V. A. Rebiazina, A. Shalaeva, M. Smirnova, Digital Transformation and Global Society Third International Conference, DTGS, 858, 386-401 (2018). (https://doi.org/10.1007/978-3-030-02843-5_31

7. V. Mkrttchian, I. Palatkin et al., Big Data and Knowledge Sharing in Virtual Organizations, 103-116. (2019) doi:10.4018/978-1-5225-7519-1.ch004.

8. Y.V. Vertakova, E.Y. Charochkina, E.D. Leontyev, Journal of Applied Engineering Science, 17, 514-517 (2019) doi:10.5937/jaes17-21360

9. K. Ala-Mutka Mapping Digital Competence: Towards a Conceptual Under standing. (2018)

10. C. Iordache, I. Marien, D. Baelden. Italian Journal of Sociology of Education, (2017).

11. E.W. Felten, M. Raj, R. Seamans, American Economic Association Papers and Proceedings. 108, 54 -57 (2018)

12. C.B. Frey, M.A. Osborne, Technological Forecasting and Social Change. 114. 254 280. (2017).

13. Y.V. Vertakova, T.A. Golovina, A.V. Polyanin Lecture Notes in Networks and Systems, 87. 856-865 (2020). DOI: 10.1007/978-3-030-29586-8_97

14. DigComp 2.1. The Digital Competence Framework for Citizens. With Eight Proficiency Levels and Examples of Use (2018)

15. The New Foundational Skills of the Digital Economy. Developing the Professionals of the Future. Available at: https://www.burn-ing-glass.com/wpontent/uploads/New_Foundational_Skills.pdf (accessed 20.01.2020).

16. R. Grundke, et al., Which skills for the digital era?: Returns to skills analysis. (Paris: OECD Publishing, 2018).

17. M. Zaki, M.H. Ismail Abdelaa, Digital Business Transformation and Strategy: What Do We Know So Far, Working Paper, (2018). 